\title{
EFFECT OF WEARING WHOLE BODY COMPRESSION GARMENTS ON CARDIOVASCULAR FUNCTION USING ECG SIGNALS
}

\author{
Lan Thi Nhu Nguyen, David Eager, Hung Tan Nguyen \\ University of Technology Sydney \\ 15 Broadway, Ultimo, New South Wave 2007, Australia \\ ThiNhuLan.Nguyen@student.uts.edu.au, David.Eager@uts.edu.au, Hung.Nguyen@uts.edu.au
}

\begin{abstract}
The purpose of this study was to examine the effects of wearing whole body compression garments (WBCGs) on cardiovascular function of running trainers. Eight nonathletes (age: 25.1 \pm 3.8 years, height: $165.9 \pm 8.3 \mathrm{~cm}$; weight: $61.4 \pm 13.7 \mathrm{~kg}$ ) performed an incremental test followed by 30 minutes running on a treadmill, from 6 $\mathrm{km} \cdot \mathrm{h}^{-1}$ to $11 \mathrm{~km} \cdot \mathrm{h}^{-1}$ with correct size-compression garments (CCGs), undersize-compression garments (UCGs) and non- compression garments (NCGs). During the exercise, electrocardiogram (ECG) signals were collected between each completed speed by wearable sensors. There was a significant difference in heart rate $(\mathrm{HR}, \mathrm{p}<0.05)$ between CCGs and NCGs from the velocity of $7 \mathrm{~km} . \mathrm{h}^{-1}$ onwards. Moreover, the group that wore UCGs has some significant effects on QT intervals and corrected QT at $10 \mathrm{~km} \cdot \mathrm{h}^{-1}$ and $11 \mathrm{~km} \cdot \mathrm{h}^{-1}(\mathrm{p}<0.05)$. The utilization of WBCGs in a running test may influence the cardiovascular function of wearers. Based on the results of longer QTc, UCGs may cause an adverse effect on performance. Essentially, CCGs should be recommended for wearing during exercise due to the effects of lower HR.
\end{abstract}

\section{KEY WORDS}

Compression garments, QT, QTc, running, heart rate, heart rate variability, performance, telehealth in sports.

\section{Introduction}

Compression garments (CGs) are becoming increasingly useful in the modern life. Existing research indicates that CGs are able to provide positive effects on the health and well-being of the wearer such as treatment of burn scar and leg pain [1, 2]. Besides, many researchers also claim that CGs are beneficial for sports activities. For example, there was a less muscle soreness, less fatigue ratings, ultrasound measure swelling and lower creatine kinase in CGs groups [3, 4]. The utilization of lower body CGs also presented a significant improvement in post-exercise recovery[5]. Consequently, compression garments are helpful as a recovery tool, providing a practical recovery strategy for team sports scenarios where an accelerated recovery due to a tight schedule is important [6].

Moreover, the effects of CGs were not only shown in recovery but also during exercise. It has been shown CGs improved repeated sprints performance [7]. The upper body CGs significantly increased upper body strength (5\% for both eccentric and concentric contraction) [8]. Some other researchers indicated that CGs increased skin temperature [8,9], enhanced oxygen consumption, O2 pulse, deoxyhemoglobin, and decreased running economy, oxyhemoglobin, tissue oxygenation index [10].

Though a lot of research demonstrated the significant benefits of CGs, in a number of studies it has been shown that there are no significant improvements in physiological responses or performance [11, 12]. The heterogeneity of the studies could be due to several different factors. For instance, there are the different garments (upper body CGs, lower body CGs, whole body CGs, CGs stockings), duration of application (during exercise, post exercise), type of activity (running, jumping, cycling), training status (hot or cold environment) [13]. One of the most important factors which may cause significant effects is the pressure [14]. Using a different size can impact on a pressure level. However, the optimal pressure, as well as the effects of various size CGs are still lacking evidence [15].

On the other hand, many tests were performed to find the underlying mechanism. For instance, a study reported that aerobically trained improvements in calf muscle pump function could create an increase in using CGs when considering venous return [16]. Similarly, CGs was able to improve blood flow [10]. It might leave to lower rating of perceived exertion influencing on performance and recovery [17]. Moreover, in the research of accuracy performance of archers, better accuracy and lower heart rate occur at the same time, based on a comparison between experienced archers and inexperienced archers [18]. However, the mechanism behind the physiological and biochemical responses of wearing CGs still remain unclear $[13,19]$.

The most prevalent cardiovascular detection systems are provided by Polar [20]. Pulse date is used as a method to process data onto these systems. However, a lot of useful information about rehabilitation services such as QT, QTc was not provided by these instruments. Some previous studies have shown a significantly increasing risk of cardiac arrhythmias associated with the prolongation of QT intervals or QTc [21]. The increasing of QT dispersion and QTc dispersion have also effected performance training [21]. Similarly, a research of MC Mandyam - 2012 claimed that longer QT interval is 
associated with an increased incident atrial fibrillation, and stroke [22]. There is a more suitable system based on ECG monitor which can be used for training program and analysis performance. The ECG systems may also provide other additional parameters which influence cardiovascular function, including RR intervals, TpTe, QRS, QT and QTc.

This study explores the underlying mechanism relating cardiovascular function using CGs. The aim of this research is to assess whether UCGs or CCGs are beneficial for non-athletes during an incremental running test on a treadmill using bio-sensors.

\section{Method}

\subsection{Participants}

Eight young and healthy non-athletes (age: 25.1 \pm 3.8 years, height: $165.9 \pm 8.3 \mathrm{~cm}$; weight: $61.4 \pm 13.7 \mathrm{~kg})$, including five men and three women, volunteered for the study. The detailed participant characteristics are shown in Table 1.

All volunteers were non-smokers, not taking any medication, and free of any cardiovascular, cerebrovascular and respiratory disease. Subjects were required to get a healthy night's sleep, not to drink caffeine or alcohol for 24 hours prior to the test and not to eat for 2 hours before the running trials. All participantswere required to complete a basic medical questionnaire before implementing the tests.

After the risks and benefits of the study had been explained, each subject signed a university informed consent document and started for the trials. The protocol has been approved by the University of Technology, Sydney Human Ethics Committee. (Approval number: UTS HREC REF NO.2014000844).

\subsection{Experimental Garments}

WBCGs, including a long-sleeved top CGs (neck and wrist to waist) and long-leg CGs (waist to ankle), were used. SportSkins Classic WBCGs (Skins, Campbelltown, NSW, Australia) were chosen in this experiment. The garments comprised of 76\% Nylon and Meryl Microfiber and 24\% Roica Spandex. CGs were made one size smaller-undersize (UCGs) and advised size-correct (CCGs) using the guidelines which provided by the manufacturer, based on subjects' stature and body mass. Two subjects wearing CGs are shown in Figure 1.

\subsection{Experimental Protocol}

Each participant performed three running sessions which are separated by at least two different days and in a randomized fashion using UCGs, CCGs or NCGs. The running was conducted in the same laboratory. Environmental temperature was stable at a range of 20$22^{\circ} \mathrm{C}$ and did not differ from the testing conditions.
Participants wore the same shoes, socks in three separate tests. All members were allowed to wear their own NCGs.

\subsection{Exercise Protocol}

Participants randomly completed three stepwise incremental tests to determine ECG signals in three different types of garments, involving UCGs, CCGs, and NCGs. Before the trials, participants were asked to complete a questionnaire about their current health. This step should take about 5 minutes.

Then, ECG electrodes were attached to the subject's upper body, based on lead II-ECG position shown in figure 2. After a rest of 10 minutes, ECG data was collected. This step was conducted to ensure heart rate returned to a normal beat.

After that, a 10 minutes warm-up at $6 \mathrm{~km} . \mathrm{h}^{-1}$ was performed on a treadmill of $0 \%$ grade. And then, participants were required to stop for about 1.5 minutes

Table 1

Participant characteristics

\begin{tabular}{|l|l|l|}
\hline & $\begin{array}{l}\text { Men }(\mathrm{n}=5) \\
\text { Mean } \pm \text { std }\end{array}$ & $\begin{array}{l}\text { Women }(\mathrm{n}=3) \\
\text { Mean } \pm \text { std }\end{array}$ \\
\hline Age $($ year) & $26.2 \pm 3.1$ & $23.3 \pm 4.9$ \\
\hline Height $(\mathrm{cm})$ & $170.6 \pm 5.5$ & $158.0 \pm 5.6$ \\
\hline Chest $(\mathrm{cm})$ & $95.9 \pm 5.5$ & $81.0 \pm 2.6$ \\
\hline Weight $(\mathrm{kg})$ & $70.6 \pm 6.0$ & $46.0 \pm 3.6$ \\
\hline Body mass index $\left(\mathrm{kg} . \mathrm{m}^{-2}\right)$ & $24.4 \pm 3.4$ & $18.4 \pm 0.9$ \\
\hline
\end{tabular}
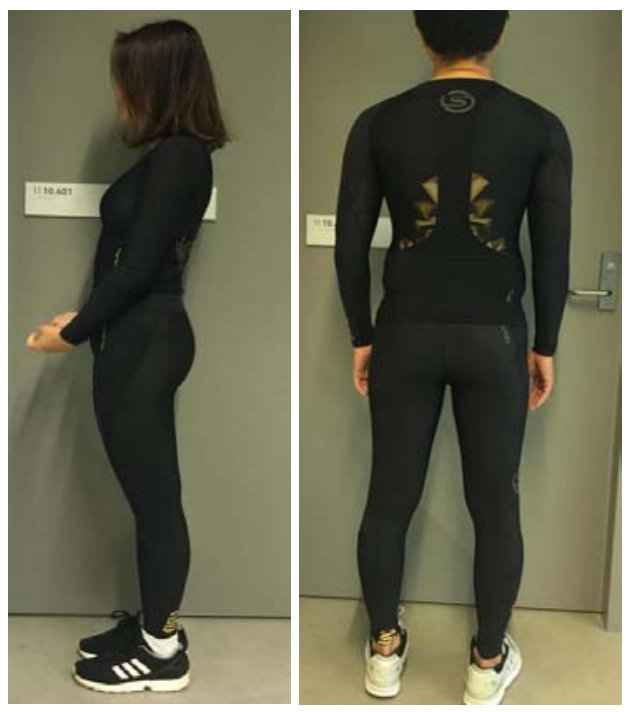

Figure 1. Subjects wear compression garments.

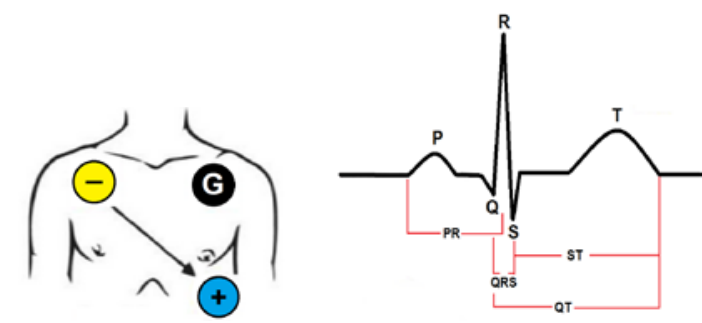

$\begin{array}{ll}\text { (a) Lead II position } & \text { (b) Detection of intervals }\end{array}$ Figure 2. ECG signals. 
for data collection. The treadmill's speed was increased by $1 \mathrm{~km} \cdot \mathrm{h}^{-1}$, and after running 2 minutes (at each velocity), participants were required to stop (1.5 minutes) for collecting data again. The process continued until reaching $11 \mathrm{~km} \cdot \mathrm{h}^{-1}$. The ECG signals were obtained as soon as participants completed each assigned speed for 1.5 minutes, using an ECG monitor (Flexcomp Infiniti Thought Technology Ltd, Canada). All tests were conducted in isolation, with only the study personnel observing each testing session.

\subsection{Statistical Analysis}

Matlab, the commercial software, was used for detecting peaks and statistical analysis. Where a significant main effect was found, a t-test was used to determine an individually significant difference. Mean values and STD (mean \pm STD) were calculated for all descriptive measures. A significant level was set at $\mathrm{p}<0.05$. All analyses were performed using Matlab version 2015b for Windows.

To compare the practical relevance and meaningfulness of various results, effect sizes (ES) were assessed using the conventional procedure proposed by Cohen $\mathrm{d}$. In the conventional manner, ES of $<0.1,0.1$ $0.3,0.3-0.5$ and $>0.5$ were regarded as trivial effect, small effect, moderate effect and large difference effect, respectively.

HRV parameters including heart rate (HR), the mean of RR interval (meanNN), number of successive RR interval pairs that differ more than 50ms (NN50), percentage of all sequential RR deviations exceeding 50 ms (pNN50), a standard deviation of RR intervals (SDNN), the rood mean square of sequential deviation (RMSSD), very low frequency (0.003-0.04 Hz - VLF), low frequency (0.04-0.15 Hz - LF), high frequency (0.15$0.4 \mathrm{~Hz}-\mathrm{HF}$ ) were analyzed by detecting RR intervals. Q, $\mathrm{S}$, Tp, Te were collected to calculated other intervals such as ST, QRS, QT and TpTe. Many different formulas can be used to indicate QTc [23]. However, Bazett was chosen as the most common formula to present many significant results [21, 22]. The Bazett's formula is shown below:

$$
Q T c=\frac{Q T}{\sqrt{R R}}
$$

All parameters of HRV were considered throughout the collected duration (90 seconds) [24]. The intervals were assessed at the immediate stop (at the first 10 seconds) for analysis.

\section{Results}

\subsection{Heart rate variability}

Heart rate variability (HRV) was analyzed within 90 seconds of the collected time. The comparison between using CCGs and NCGs indicated some significant

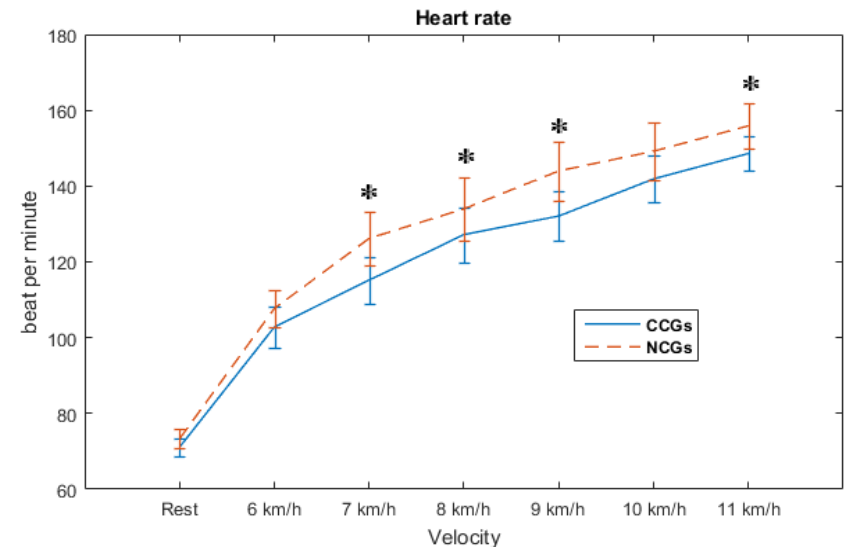

Figure 3. A comparison of heart rate between using correct size- compression garments (CCGs) and non-compression garments (NCGs).

*Significant difference compared with NCGs $(\mathrm{p}<0.05)$.

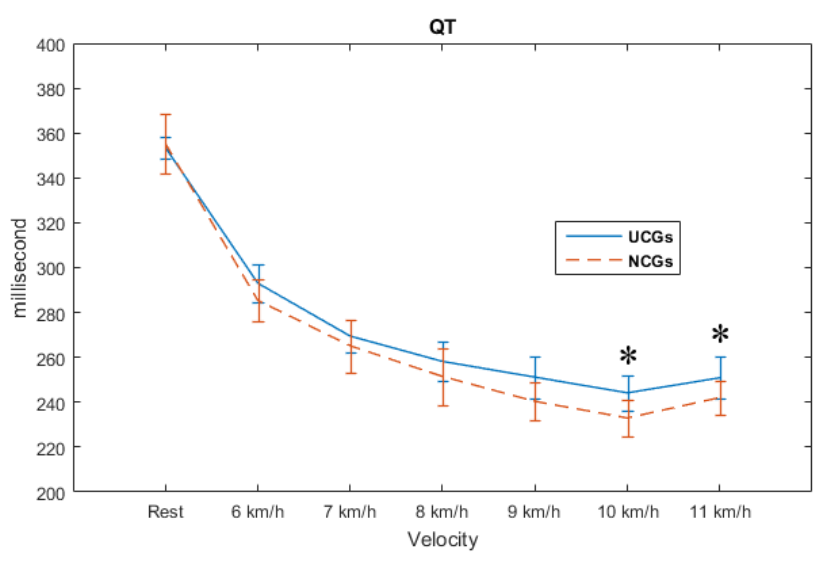

Figure 4. A comparison of QT intervals between using undersize-compression garments (UCGs) and noncompression garments (NCGs).

*Significant difference compared with NCGs $(\mathrm{p}<0.05)$.

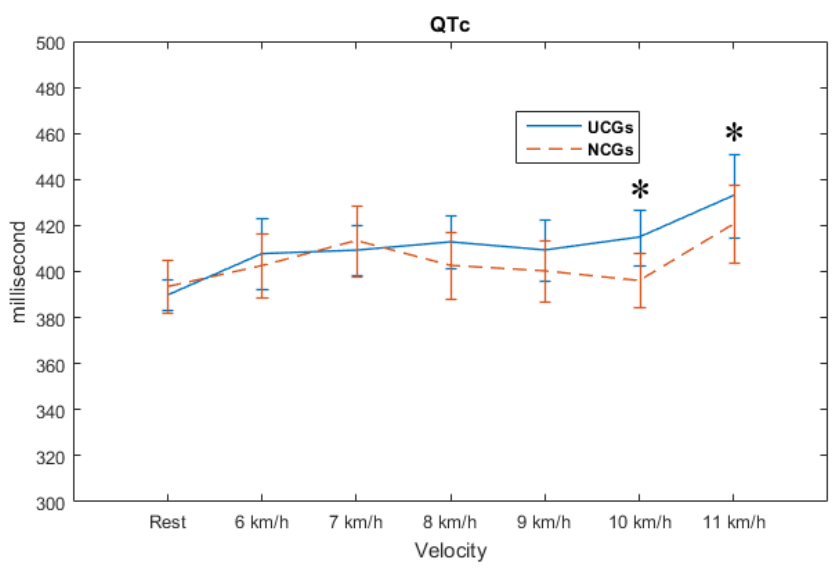

Figure 5. A comparison of corrected QT (QTc) between using undersize-compression garments (UCGs) and noncompression garments (NCGs).

*Significant difference compared with NCGs $(\mathrm{p}<0.05)$. 
Table 2

Response of heart rate variability in correct size-compression garments and non-compression garments

\begin{tabular}{|c|c|c|c|c|c|c|c|c|c|c|c|}
\hline & & HR & SDNN & RMSSD & NN50 & PNN50 & SD1 & SD2 & VLF & LF & $\mathrm{HF}$ \\
\hline \multirow[t]{2}{*}{ Rest } & $\mathrm{C}$ & $71.1 \pm 6.7$ & $61.9 \pm 31.3$ & $61.6 \pm 31.2$ & $29.3 \pm 20.7$ & $0.3 \pm 0.2$ & $38.1 \pm 21.7$ & $78.2 \pm 40.3$ & $19.1 \pm 15.9$ & $49.8 \pm 16.4$ & $25.5 \pm 11.1$ \\
\hline & $\mathrm{N}$ & $73.3 \pm 7.2$ & $48.2 \pm 15.4$ & $48.0 \pm 15.3$ & $16.9 \pm 13.6$ & $0.1 \pm 0.1$ & $25.5 \pm 11.0$ & $62.1 \pm 20.4$ & $32.5 \pm 28.2$ & $35.5 \pm 20.1$ & $30.2 \pm 20.0$ \\
\hline \multirow{2}{*}{$\begin{array}{l}6 \\
\mathrm{~km} / \mathrm{h}\end{array}$} & $\mathrm{C}$ & $102.8 \pm 15.0$ & $57.0 \pm 19.9$ & $56.8 \pm 19.8$ & $7.5 \pm 9.8$ & $0.1 \pm 0.1$ & $15.0 \pm 8.6$ & $78.9 \pm 27.2$ & $43.2 \pm 18.2$ & $42.4 \pm 15.2$ & $12.7 \pm 8.6$ \\
\hline & $\mathrm{N}$ & $107.7 \pm 13.7$ & $51.5 \pm 27.0$ & $51.4 \pm 26.9$ & $3.9 \pm 4.6$ & $0 \pm 0$ & $11.8 \pm 5.7$ & $71.4 \pm 37.9$ & $49.2 \pm 14.0$ & $31.5 \pm 7.7$ & $17.5 \pm 8.8$ \\
\hline \multirow{2}{*}{$\begin{array}{l}7 \\
\mathrm{~km} / \mathrm{h}\end{array}$} & $\mathrm{C}$ & $115.2 \pm 17.5^{* *}$ & $78.1 \pm 22.0^{* *}$ & $77.9 \pm 21.9^{* *}$ & $7.9 \pm 12.8$ & $0.1 \pm 0.1$ & $13.4 \pm 10.4$ & $108.8 \pm 29.7^{* *}$ & $67.4 \pm 23.4$ & $21.2 \pm 17.6$ & $8.6 \pm 7.7^{*}$ \\
\hline & $\mathrm{N}$ & $126.2 \pm 19.9$ & $60.6 \pm 19.5$ & $60.4 \pm 19.4$ & $5.0 \pm 6.6$ & $0 \pm 0$ & $14.0 \pm 11.4$ & $83.4 \pm 27.2$ & $35.4 \pm 28.0$ & $26.0 \pm 12.5$ & $24.5 \pm 14.1$ \\
\hline \multirow{2}{*}{$\begin{array}{l}8 \\
\mathrm{~km} / \mathrm{h}\end{array}$} & $\mathrm{C}$ & $127.2 \pm 20.2^{*}$ & $66.8 \pm 31.1^{*}$ & $66.6 \pm 30.9 *$ & $4.5 \pm 9.9$ & $0 \pm 0.1$ & $10.3 \pm 12.2$ & $93.5 \pm 42.8^{*}$ & $37.7 \pm 28.2$ & $26.0 \pm 10.6$ & $26.9 \pm 18.2$ \\
\hline & $\mathrm{N}$ & $134.0 \pm 23.2$ & $49.2 \pm 14.6$ & $49.1 \pm 14.5$ & $2.4 \pm 3.3$ & $0 \pm 0$ & $6.9 \pm 5.0$ & $68.9 \pm 20.2$ & $30.4 \pm 28.0$ & $25.2 \pm 10.7$ & $32.6 \pm 18.4$ \\
\hline \multirow{2}{*}{$\begin{array}{l}99 \\
\mathrm{~km} / \mathrm{h}\end{array}$} & $\mathrm{C}$ & $132.1 \pm 18.3^{*}$ & $63.1 \pm 30.2$ & $63.0 \pm 30.1$ & $4.9 \pm 9.5$ & $0 \pm 0.1$ & $9.8 \pm 11.1$ & $88.4 \pm 41.8$ & $41.1 \pm 22.7$ & $27.2 \pm 14.6$ & $25.1 \pm 18.6$ \\
\hline & $\mathrm{N}$ & $144.0 \pm 22.2$ & $46.4 \pm 16.1$ & $46.3 \pm 16.0$ & $0.5 \pm 1.4$ & $0 \pm 0$ & $4.5 \pm 3.1$ & $65.3 \pm 22.6$ & $29.2 \pm 27.4$ & $26.6 \pm 15.4$ & $31.6 \pm 16.2$ \\
\hline \multirow{2}{*}{$\begin{array}{l}10 \\
\mathrm{~km} / \mathrm{h}\end{array}$} & $\mathrm{C}$ & $141.9 \pm 17.9$ & $60.2 \pm 28.6$ & $60.1 \pm 28.5$ & $2.1 \pm 4.4$ & $0 \pm 0$ & $13.5 \pm 22.5$ & $82.3 \pm 37.8$ & $40.5 \pm 32.8$ & $16.6 \pm 8.2$ & $33.5 \pm 21.8$ \\
\hline & $\mathrm{N}$ & $149.2 \pm 21.4$ & $46.1 \pm 13.8$ & $46.0 \pm 13.8$ & $0.3 \pm 0.7$ & $0 \pm 0$ & $4.1 \pm 2.3$ & $64.8 \pm 19.4$ & $29.2 \pm 33.2$ & $22.3 \pm 12.0$ & $37.4 \pm 18.1$ \\
\hline \multirow{2}{*}{$\begin{array}{l}11 \\
\mathrm{~km} / \mathrm{h}\end{array}$} & $\mathrm{C}$ & $148.6 \pm 12.4^{*}$ & $50.7 \pm 22.4$ & $50.6 \pm 22.3$ & $1.3 \pm 3.2$ & $0 \pm 0$ & $4.7 \pm 4.5$ & $71.3 \pm 31.5$ & $35.1 \pm 29.7$ & $24.0 \pm 8.7$ & $26.0 \pm 16.7$ \\
\hline & $\mathrm{N}$ & $155.9 \pm 17.1$ & $42.3 \pm 13.4$ & $42.2 \pm 13.4$ & $0.6 \pm 1.8$ & $0 \pm 0$ & $3.8 \pm 2.5$ & $59.5 \pm 18.9$ & $34.2 \pm 35.3$ & $22.6 \pm 12.2$ & $29.4 \pm 22.1$ \\
\hline
\end{tabular}

C-Correct size-compression garments; N-Non compression garments;

$* * * \mathrm{p}<0.001,{ }^{* *} \mathrm{p}<0.01,{ }^{*} \mathrm{p}<0.05$.

Table 3

Response of heart rate variability in undersize-compression garments and non-compression garments

\begin{tabular}{|c|c|c|c|c|c|c|c|c|c|c|c|}
\hline & & HR & SDNN & RMSSD & NN50 & PNN50 & SD1 & SD2 & VLF & LF & HF \\
\hline \multirow[t]{2}{*}{ Rest } & $\mathrm{U}$ & $72.3 \pm 4.3$ & $56.2 \pm 32.6$ & $56.0 \pm 32.4$ & $19.4 \pm 17.4$ & $0.2 \pm 0.2$ & $29.9 \pm 20.9$ & $73.3 \pm 42.0$ & $16.2 \pm 12.5$ & $54.3 \pm 9.5$ & $27.7 \pm 11.9$ \\
\hline & $\mathrm{N}$ & $73.3 \pm 7.2$ & $48.2 \pm 15.4$ & $48.0 \pm 15.3$ & $16.9 \pm 13.6$ & $0.1 \pm 0.1$ & $25.5 \pm 11.0$ & $62.1 \pm 20.4$ & $32.5 \pm 28.2$ & $35.5 \pm 20.1$ & $30.2 \pm 20.0$ \\
\hline \multirow{2}{*}{$\begin{array}{l}6 \\
\mathrm{~km} / \mathrm{h}\end{array}$} & $\mathrm{U}$ & $99.9 \pm 9.6^{*}$ & $58.8 \pm 23.4$ & $58.6 \pm 23.3$ & $10.3 \pm 12.1$ & $0.1 \pm 0.1$ & $16.1 \pm 9.0$ & $81.1 \pm 32.2$ & $47.9 \pm 23.0$ & $35.5 \pm 18.8$ & $11.9 \pm 8.3$ \\
\hline & $\mathrm{N}$ & $107.7 \pm 13.7$ & $51.5 \pm 27.0$ & $51.4 \pm 26.9$ & $3.9 \pm 4.6$ & $0 \pm 0$ & $11.8 \pm 5.7$ & $71.4 \pm 37.9$ & $49.2 \pm 14.0$ & $31.5 \pm 7.7$ & $17.5 \pm 8.8$ \\
\hline \multirow{2}{*}{$\begin{array}{l}7 \\
\mathrm{~km} / \mathrm{h}\end{array}$} & $\mathrm{U}$ & $117.9 \pm 19.3^{*}$ & $65.4 \pm 30.8$ & $65.2 \pm 30.7$ & $6.9 \pm 12.4$ & $0 \pm 0.1$ & $11.3 \pm 11.3$ & $91.0 \pm 42.7$ & $53.8 \pm 31.7$ & $28.0 \pm 21.2$ & $11.5 \pm 10.0$ \\
\hline & $\mathrm{N}$ & $126.2 \pm 19.9$ & $60.6 \pm 19.5$ & $60.4 \pm 19.4$ & $5.0 \pm 6.6$ & $0 \pm 0$ & $14.0 \pm 11.4$ & $83.4 \pm 27.2$ & $35.4 \pm 28.0$ & $26.0 \pm 12.5$ & $24.5 \pm 14.1$ \\
\hline \multirow{2}{*}{$\begin{array}{l}8 \\
\mathrm{~km} / \mathrm{h}\end{array}$} & $\mathrm{U}$ & $127.8 \pm 22.0$ & $64.4 \pm 31.9$ & $64.2 \pm 31.8$ & $4.4 \pm 9.9$ & $0 \pm 0.1$ & $9.9 \pm 12.5$ & $90.0 \pm 43.9$ & $34.4 \pm 23.1$ & $29.2 \pm 8.0$ & $29.3 \pm 16.1$ \\
\hline & $\mathrm{N}$ & $134.0 \pm 23.2$ & $49.2 \pm 14.6$ & $49.1 \pm 14.5$ & $2.4 \pm 3.3$ & $0 \pm 0$ & $6.9 \pm 5.0$ & $68.9 \pm 20.2$ & $30.4 \pm 28.0$ & $25.2 \pm 10.7$ & $32.6 \pm 18.4$ \\
\hline \multirow{2}{*}{$\begin{array}{l}9 \\
\mathrm{~km} / \mathrm{h}\end{array}$} & $\mathrm{U}$ & $135.4 \pm 21.6$ & $62.5 \pm 29.5$ & $62.3 \pm 29.4$ & $4.8 \pm 9.2$ & $0 \pm 0.1$ & $9.4 \pm 11.6$ & $87.4 \pm 40.7$ & $40.4 \pm 28.2$ & $24.2 \pm 17.6$ & $24.7 \pm 16.9$ \\
\hline & $\mathrm{N}$ & $144.0 \pm 22.2$ & $46.4 \pm 16.1$ & $46.3 \pm 16.0$ & $0.5 \pm 1.4$ & $0 \pm 0$ & $4.5 \pm 3.1$ & $65.3 \pm 22.6$ & $29.2 \pm 27.4$ & $26.6 \pm 15.4$ & $31.6 \pm 16.2$ \\
\hline \multirow{2}{*}{$\begin{array}{l}10 \\
\mathrm{~km} / \mathrm{h}\end{array}$} & $\mathrm{U}$ & $146.1 \pm 22.3$ & $56.5 \pm 28.0$ & $56.4 \pm 27.9$ & $1.8 \pm 4.2$ & $0 \pm 0$ & $7.4 \pm 7.8$ & $79.1 \pm 39.1$ & $31.3 \pm 35.8$ & $18.0 \pm 9.7$ & $37.9 \pm 22.4$ \\
\hline & $\mathrm{N}$ & $149.2 \pm 21.4$ & $46.1 \pm 13.8$ & $46.0 \pm 13.8$ & $0.3 \pm 0.7$ & $0 \pm 0$ & $4.1 \pm 2.3$ & $64.8 \pm 19.4$ & $29.2 \pm 33.2$ & $22.3 \pm 12.0$ & $37.4 \pm 18.1$ \\
\hline \multirow{2}{*}{$\begin{array}{l}11 \\
\mathrm{~km} / \mathrm{h}\end{array}$} & $\mathrm{U}$ & $152.7 \pm 17.1^{*}$ & $49.5 \pm 26.0$ & $49.4 \pm 25.9$ & & $0 \pm 0$ & $11.2 \pm 19.8$ & $67.2 \pm 35.0$ & $40.9 \pm 30.7$ & $20.2 \pm 10.7$ & $28.5 \pm 18.7$ \\
\hline & $\mathrm{N}$ & $155.9 \pm 17.1$ & $42.3 \pm 13.4$ & $42.2 \pm 13.4$ & $0.6 \pm 1.8$ & $0 \pm 0$ & $3.8 \pm 2.5$ & $59.5 \pm 18.9$ & $34.2 \pm 35.3$ & $22.6 \pm 12.2$ & $29.4 \pm 22.1$ \\
\hline
\end{tabular}

U-Undersize-compression garment; N-Non compression garments;

$* * * \mathrm{p}<0.001,{ }^{* *} \mathrm{p}<0.01,{ }^{*} \mathrm{p}<0.05$.

Table 4

QT and QTc response when wearing correct sizecompression garments and non-compression garments

\begin{tabular}{|c|c|c|c|c|c|}
\hline \multicolumn{2}{|c|}{ Variable } & QT (ms) & $\mathrm{p}$ & QTc (ms) & p, \\
\hline \multirow[t]{2}{*}{ Rest } & $\mathrm{C}$ & $357.1 \pm 21.4$ & \multirow{2}{*}{$\begin{array}{l}0.8476, \\
0.0595\end{array}$} & $383.4 \pm 23.0$ & \multirow{2}{*}{$\begin{array}{l}0.3023 \text {, } \\
0.3591\end{array}$} \\
\hline & $\mathrm{N}$ & $355.3 \pm 38.2$ & & $393.5 \pm 32.3$ & \\
\hline \multirow{2}{*}{$\begin{array}{l}6 \\
\mathrm{~km} / \mathrm{h}\end{array}$} & $\mathrm{C}$ & $290.9 \pm 28.2$ & \multirow{2}{*}{$\begin{array}{l}0.3622, \\
0.2021\end{array}$} & $401.3 \pm 39.7$ & \multirow{2}{*}{$\begin{array}{l}0.9127, \\
0.0316 \\
\end{array}$} \\
\hline & $\mathrm{N}$ & $285.4 \pm 26.5$ & & $402.6 \pm 39.7$ & \\
\hline \multirow{2}{*}{$\begin{array}{l}7 \\
\mathrm{~km} / \mathrm{h}\end{array}$} & $\mathrm{C}$ & $276.0 \pm 27.3$ & \multirow{2}{*}{$\begin{array}{l}0.1115, \\
0.3557\end{array}$} & $415.8 \pm 47.5$ & \multirow{2}{*}{$\begin{array}{l}0.7851, \\
0.0490\end{array}$} \\
\hline & $\mathrm{N}$ & $265.2 \pm 33.4$ & & $413.5 \pm 43.7$ & \\
\hline \multirow{2}{*}{$\begin{array}{l}8 \\
\mathrm{~km} / \mathrm{h}\end{array}$} & $\mathrm{C}$ & $267.6 \pm 48.8$ & \multirow{2}{*}{$\begin{array}{l}0.2773, \\
0.3777\end{array}$} & $424.4 \pm 83.8$ & \multirow{2}{*}{$\begin{array}{l}0.3655 \\
0.3286\end{array}$} \\
\hline & $\mathrm{N}$ & $251.5 \pm 35.6$ & & $402.7 \pm 41.4$ & \\
\hline \multirow{2}{*}{$\begin{array}{l}9 \\
\mathrm{~km} / \mathrm{h}\end{array}$} & C & $252.5 \pm 21.4$ & \multirow{2}{*}{$\begin{array}{l}\text { 0.0949, } \\
0.5361\end{array}$} & $411.1 \pm 31.6$ & \multirow{2}{*}{$\begin{array}{l}0.2817, \\
0.3159\end{array}$} \\
\hline & $\mathrm{N}$ & $240.4 \pm 23.7$ & & $400.3 \pm 36.6$ & \\
\hline \multirow{2}{*}{$\begin{array}{l}10 \\
\mathrm{~km} / \mathrm{h}\end{array}$} & C & $239.8 \pm 24.3$ & \multirow{2}{*}{$\begin{array}{l}0.1529, \\
0.2912\end{array}$} & $404.0 \pm 34.9$ & \multirow{2}{*}{$\begin{array}{l}0.2839, \\
0.2305\end{array}$} \\
\hline & $\mathrm{N}$ & $233.0 \pm 22.6$ & & $396.1 \pm 33.3$ & \\
\hline \multirow{2}{*}{$\begin{array}{l}11 \\
\mathrm{~km} / \mathrm{h}\end{array}$} & C & $248.4 \pm 31.4$ & \multirow{2}{*}{$\begin{array}{l}\text { 0.3104, } \\
0.2325\end{array}$} & $427.4 \pm 57.9$ & \multirow{2}{*}{$\begin{array}{l}0.5589, \\
0.1264\end{array}$} \\
\hline & $\mathrm{N}$ & $242.1 \pm 21.8$ & & $420.7 \pm 48.0$ & \\
\hline
\end{tabular}

C-Correct size compression garments; N-Non compression garments; QT-QT intervals; QTc-Corrected QT; $* * * \mathrm{p}<0.001,{ }^{* *} \mathrm{p}<0.01,{ }^{*} \mathrm{p}<0.05$.
Table 5

QT and QTc response when wearing undersizecompression garments and non-compression garments

\begin{tabular}{|c|c|c|c|c|c|}
\hline \multicolumn{2}{|c|}{ Variable } & QT (ms) & $\mathrm{p}$ & QTc (ms) & $\mathrm{p}$ \\
\hline \multirow{2}{*}{ Rest } & $\overline{\mathrm{U}}$ & $353.5 \pm 13.9$ & \multirow{2}{*}{$\begin{array}{l}\text { 0.8940, } \\
0.062\end{array}$} & $389.8 \pm 18.8$ & \multirow{2}{*}{$\begin{array}{l}0.6546, \\
0.1395\end{array}$} \\
\hline & $\mathrm{N}$ & $355.3 \pm 38.2$ & & $393.5 \pm 32.3$ & \\
\hline \multirow{2}{*}{$\begin{array}{l}6 \\
\mathrm{~km} / \mathrm{h}\end{array}$} & U & $293.2 \pm 24.6$ & \multirow{2}{*}{$\begin{array}{l}0.2647, \\
0.3036\end{array}$} & $407.8 \pm 44.2$ & \multirow{2}{*}{$\begin{array}{l}0.7001, \\
0.1234\end{array}$} \\
\hline & $\mathrm{N}$ & $285.4 \pm 26.5$ & & $402.6 \pm 39.7$ & \\
\hline \multirow{2}{*}{$\begin{array}{l}7 \\
\mathrm{~km} / \mathrm{h}\end{array}$} & $\mathrm{U}$ & $269.5 \pm 21.1$ & \multirow{2}{*}{$\begin{array}{l}0.5327, \\
0.1558\end{array}$} & $409.3 \pm 30.5$ & \multirow{2}{*}{$\begin{array}{l}0.6166, \\
0.1117\end{array}$} \\
\hline & $\mathrm{N}$ & $265.2 \pm 33.4$ & & $413.5 \pm 43.7$ & \\
\hline \multirow{2}{*}{$\begin{array}{l}8 \\
\mathrm{~km} / \mathrm{h}\end{array}$} & $\mathrm{U}$ & $258.2 \pm 24.6$ & \multirow{2}{*}{$\begin{array}{l}0.2518, \\
0.2193\end{array}$} & $412.9 \pm 32.0$ & \multirow{2}{*}{$\begin{array}{l}0.1565, \\
0.2767\end{array}$} \\
\hline & $\mathrm{N}$ & $251.5 \pm 35.6$ & & $402.7 \pm 41.4$ & \\
\hline \multirow{2}{*}{$\begin{array}{l}9 \\
\mathrm{~km} / \mathrm{h}\end{array}$} & $\mathrm{U}$ & $251.2 \pm 26.4$ & \multirow{2}{*}{$\begin{array}{l}0.1247, \\
0.4317\end{array}$} & $409.4 \pm 37.0$ & \multirow{2}{*}{$\begin{array}{l}0.454, \\
0.2477\end{array}$} \\
\hline & $\mathrm{N}$ & $240.4 \pm 23.7$ & & $400.3 \pm 36.6$ & \\
\hline \multirow{2}{*}{$\begin{array}{l}10 \\
\mathrm{~km} / \mathrm{h}\end{array}$} & $\mathrm{U}$ & $244.2 \pm 22.0$ & \multirow{2}{*}{$\begin{array}{l}0.0242, * \\
0.5008\end{array}$} & $415.0 \pm 34.0$ & \multirow{2}{*}{$\begin{array}{l}0.0038^{*} \\
* \\
0.5611\end{array}$} \\
\hline & $\mathrm{N}$ & $233.0 \pm 22.6$ & & $396.1 \pm 33.3$ & \\
\hline \multirow{2}{*}{$\begin{array}{l}11 \\
\mathrm{~km} / \mathrm{h}\end{array}$} & $\mathrm{U}$ & $250.9 \pm 26.3$ & \multirow{2}{*}{$\begin{array}{l}0.0218, * \\
0.367\end{array}$} & $433.1 \pm 50.9$ & \multirow{2}{*}{$\begin{array}{l}0.0028 * \\
* \\
0.2518\end{array}$} \\
\hline & $\mathrm{N}$ & $242.1 \pm 21.8$ & & $420.7 \pm 48.0$ & \\
\hline
\end{tabular}

U-Undersize compression garments; N-Non compression garments; QT-QT intervals; QTc-Corrected QT; 


$$
{ }^{* * *} \mathrm{p}<0.001,{ }^{* *} \mathrm{p}<0.01,{ }^{*} \mathrm{p}<0.05 .
$$

differences in $\mathrm{HR}$ after running at $7 \mathrm{~km} \cdot \mathrm{h}^{-1}(\mathrm{p}=0.0033$, $\mathrm{ES}=0.5870)$, at $8 \mathrm{~km} \cdot \mathrm{h}^{-1}(\mathrm{p}=0.0473, \mathrm{ES}=0.3133)$, at 9 $\mathrm{km} \cdot \mathrm{h}^{-1}(\mathrm{p}=0.0251, \mathrm{ES}=0.5864)$, at $10 \mathrm{~km} \cdot \mathrm{h}^{-1}(\mathrm{p}=0.0804$, $\mathrm{ES}=0.3722)$ and at $11 \mathrm{~km} \cdot \mathrm{h}^{-1}(\mathrm{p}=0.0165$, $\mathrm{ES}=0.4887)$, as shown in Figure 3. Similarly, UCGs represented a significant difference in HR, compared with NCGs at 6 km.h-1 with $\mathrm{p}=0.0162$, ES $=0.6575$, at $7 \mathrm{~km} . \mathrm{h}-1$ with $\mathrm{p}=0.0491, \mathrm{ES}=0.4243$ and at $11 \mathrm{~km} . \mathrm{h}-1$ with $\mathrm{p}=0.0490$, $\mathrm{ES}=0.1865$.

In a similar manner, the difference between CCGs and NCGs revealed some remarkable results in SDNN and RMSSD. The parameters of SDNN and RMSSD presented a significant alteration at the same velocity of 7 $\mathrm{km} \cdot \mathrm{h}^{-1}$ with $\mathrm{p}=0.003, \mathrm{ES}=0.8444$, and $\mathrm{p}=0.003$, $\mathrm{ES}=0.8445$, respectively. At $8 \mathrm{~km} \cdot \mathrm{h}^{-1}$, there was $\mathrm{p}=0.0497, \mathrm{ES}=0.7254$ of SDNN and $\mathrm{p}=0.0496$, $\mathrm{ES}=0.7256$ of RMSSD. However, at three end steps of the exercise, both SDNN and RMSSD got p-value $>0.05$, but ES-values were still moderate and large. For instance, SDNN demonstrated ES=0.6909 at $9 \mathrm{~km} \cdot \mathrm{h}^{-1}$, ES=0.6273 at $10 \mathrm{~km} \cdot \mathrm{h}^{-1}$ and $\mathrm{ES}=0.4532$ at $11 \mathrm{~km} \cdot \mathrm{h}^{-1}$. Similarly, RMSSD pointed to some large effect sizes of $E S=0.6910$ at $9 \mathrm{~km} \cdot \mathrm{h}^{-1}, \mathrm{ES}=0.6295$ at $10 \mathrm{~km} \cdot \mathrm{h}^{-1}$, and $\mathrm{ES}=0.4534$ at $11 \mathrm{~km} \cdot \mathrm{h}^{-1}$. However, there was no significant difference in wearing UCGs on SDNN and RMSSD during the exercise compared to the case where NCGs are worn.

Other HRV parameters such as NN50, pNN50, and SD1 demonstrated non-significant difference during the running test $(p>0.05)$ in both size-groups of garments compared with NCGs. In the comparison between CCGs and NCGs of SD2, the analyzed results showed statistical significance at the velocity of $7 \mathrm{~km} \cdot \mathrm{h}^{-1}$ with $\mathrm{p}=0.0018$, $\mathrm{ES}=0.8884$, and $\mathrm{p}=0.048$, ES $=0.7332$ at $8 \mathrm{~km} . \mathrm{h}^{-1}$. In spite of getting $\mathrm{p}$-value $>0.05$, ES of SD2 continued to show a large attention at $9 \mathrm{~km} \cdot \mathrm{h}^{-1}, 10 \mathrm{~km} \cdot \mathrm{h}^{-1}$ and $11 \mathrm{~km} \cdot \mathrm{h}^{-1}$ with $\mathrm{ES}=0.6888, \mathrm{ES}=0.5828$ and $\mathrm{ES}=0.4544$, respectively. In contrast, there was an insignificant difference in SD2 between using UCGs and NCGs during the running test.

All frequency parameters including VLF, LF, HF, LFHF have observed no alteration $(p>0.05)$ at all velocities in CCGs and UCGs compared with NCGs. With the exception of the speed of $7 \mathrm{~km} . \mathrm{h}^{-1}$, HF indicated a statistical significance with $p=0.0415, E S=1.400$ in the group using correct size, compared with the group wearing NCGs. The results of HRV are shown in Table 2 and Table 3.

\subsection{Intervals}

Intervals were calculated at the first 10 seconds of the collected time for each velocity. Interestingly, the analysis illustrated a significant difference in QT and QTc towards the end of the test in the group of wearing under-size, compared with the group of non-wearing compression garments, according to Figure 4 and Figure 5 . At $10 \mathrm{~km} . \mathrm{h}^{-}$ 1, QT revealed a p-value $=0.0242$, ES $=0.5008$ and QTc got $\mathrm{p}=0.0038$, $\mathrm{ES}=0.5611$. Similarly, there is a significant statistically difference in QT and QTc at $11 \mathrm{~km} \cdot \mathrm{h}^{-1}$ with $\mathrm{p}=0.0218$, $\mathrm{ES}=0.3670$, and $\mathrm{p}=0.0028$, $\mathrm{ES}=0.2518$, respectively. All other parameters demonstrated no difference, including TpTe, QRS at all velocities. The UCGs group identified a no different result, compared with NCGs-group in all indicators during the exercise. The detailed results are shown in Table 4 and Table 5.

Furthermore, QTc was also re-calculated by two different formulas of Fridericia $\left(\mathrm{QTc}=\mathrm{QT} / \mathrm{RR}^{1 / 3}\right)$ and Framingham $(\mathrm{QTc}=\mathrm{Qt}+0.154(1-\mathrm{RR}))$ in order to confirming the analyzed results. The significant difference in QTc still appeared with the other formulas. For example in Fridericia formula, QTc showed the significant variety of $\mathrm{p}=0.0072$ and $\mathrm{p}=0.0061$ when comparing UCGs and NCGs at $10 \mathrm{~km} \cdot \mathrm{h}^{-1}$ and $11 \mathrm{~km} \cdot \mathrm{h}^{-1}$, respectively. Similarly, the results of QTc in the group using UCGs were $\mathrm{p}=0.012$ at $10 \mathrm{~km} \cdot \mathrm{h}^{-1}$ and $\mathrm{p}=0.0094$ at $11 \mathrm{~km} . \mathrm{h}^{-1}$, compared with NCGs, using the Framingham formula.

\section{Discussion}

The present investigation revealed a significantly lower $\mathrm{HR}$ in the group of CCGs compared with NCGs (p < 0.05 ) after reaching high velocity. The result is consistent with some previous studies. For example, the results of no difference at low intensity (8-10 km.h $\left.\mathrm{h}^{-1}\right)$ and a significantly lower HR at high intensity $\left(12-18 \mathrm{~km} . \mathrm{h}^{-1}\right)$ is consistent with a previous publication relating to welltrained runners using CGs [10]. Currently, in this study the velocity of up to $11 \mathrm{~km} \cdot \mathrm{h}^{-1}$ is considered because participants were all non-trained runners. Therefore, the results were shown at a lower speed $\left(7 \mathrm{~km} \cdot \mathrm{h}^{-1}\right)$. Similarly, a lower HR was indicated in incremental cycling bouts on a cycle ergometer in the group of CGs, compared with using above-knee cycling shorts [25].

The lower HR may relate to the lower stress, based on the results of an archery-competition [18]. Moreover, a study on eleven pitchers and ten golfers showed improvement of performance by using CGs, including the increase in fastball accuracy, driving accuracy, shot accuracy and chipping accuracy [26].

All other HRV parameters investigated no significant variety such as NN50, pNN50, SDNN, RMSSD, SD1, SD2, ULF, VLF, LF, HF in UCGs compared with NCGs $(\mathrm{p}>0.05)$. The analysis can be claimed that the using of CGs (smaller size and advised garments) may have no significant effect on HRV, except the lower HR, in the running exercise on a treadmill up to $11 \mathrm{~km} . \mathrm{h}^{-1}$.

When calculating ECG signals, the utilization of UCGs presented the significantly longer results of QT and QTc after running at $10 \mathrm{~km} \cdot \mathrm{h}^{-1}$ and $11 \mathrm{~km} \cdot \mathrm{h}^{-1}(\mathrm{p}<0.05)$. During the experience, these indicators were not showing any significant effects in two different sizes of CGs compared with NCGs.

Importantly, the previous research concluded the normal corrected QTc is less than 440 (ms) in adult male and less than 450 (ms) in adult female, suggested by Bazett [23]. The corrected QT represented an increase 
during the test. These values may get the prolonged QTc when reaching the higher speed. Based on the mentioned effects of longer QT and longer QTc in high velocity, using UCGs may cause an adverse effect on wearers due to some evidence of previous research [21,22].

\section{Conclusion}

The present study has indicated that wearing WBCGs may affect cardiovascular function. With the results of longer QTc, undersize-compression garments may cause adverse effects on wearers' performance. The application of correct size-compression garments should be recommended for exercise by the efficacy of lower HR. Further research is required to examine physiological and physical effects of compression garments in sports according to different type of exercises or other measurement methods.

\section{References}

[1] K. Rappoport, Dental and skeletal changes during pressure garment use in facial burns: a systematic review. Burns, vol. 34, 2008, 18-23.

[2] S. Lambert, The effect of graduated compression garments (Skins ${ }^{\mathrm{TM}}$ ) on perceived leg pain, leg power and alertness following elite AFL matches. Journal of Science and Medicine in Sport, vol. 9, 2006, 18.

[3] Marques-Jimenez, Are compression garments effective for the recovery of exercise-induced muscle damage. Physiology \& Behavior, vol. 153, 2016, 133-148. [4] J. A. Hill, Influence of Compression Garments on Recovery After Marathon Running. Journal of Strength \& Conditioning Research, vol. 28, 2014, 2228-2235.

[5] C. L. Pruscino, Effects of compression garments on recovery following intermittent exercise. European journal of applied physiology, vol. 113, 2013, 1585-1596.

[6] E. Gallaher, Compression garments enhance recovery following Australian Football training. Journal of Science and Medicine in Sport, vol. 13, Supplement 1, 12/ 2010, e39-e40.

[7] D.-P. Born, A novel compression garment with adhesive silicone stripes improves repeated sprint performance-a multi-experimental approach on the underlying mechanisms. BMC Sports Science, Medicine and Rehabilitation, vol. 6, 2014, 21-30.

[8] S. Morrison, Compression garments do not alter cerebrovascular responses to orthostatic stress after mild passive heating. Scandinavian journal of medicine \& science in sports, vol. 24, 2012, 291-300.

[9] B. Doan, Evaluation of a lower-body compression garment. Journal of sports sciences, vol. 21, 2003, 601610.

[10] B. J. Dascombe, The effects of wearing undersized lower-body compression garments on endurance running performance. Int J Sports Physiol Perform, vol. 6 , 2011, 160-173.
[11] B. Dascombe, No effect of upper body compression garments in elite flat-water kayakers. European journal of sport science, vol. 13, 2013, 341-349.

[12] Brighenti, Effect of new type of compreesion garments on sub-maximal and maximal cycling performance in the heat. Sport Sciences for Health, vol.9, 2013, 127-131.

[13] J. Hill, Do they really work? Sport \& Exercise Scientist, Winter2012 2012, 18-19.

[14] S. Perrey, Compression garments: Evidence for their physiological effects (P208). in The Engineering of Sport 7, ed: Springer, 2008, 319-328.

[15] M. B. A. MacRae, Compression garments and exercise. Sports medicine, vol. 41, 2011, 815-843.

[16] B. C. Rider, Effect of Compression Stockings on Physiological Responses and Running Performance in Division III Collegiate Cross-Country Runners During a Maximal Treadmill Test. Journal of Strength \& Conditioning Research, vol. 28, 2014, 1732-1738.

[17] J. A. Faulkner, Effect of Lower-Limb Compression Clothing on 400-m Sprint Performance. Journal of Strength \& Conditioning Research, vol. 27, 2013, 669676.

[18] F. Clemente, Study of the Heart Rate and Accuracy Performance of Archers. Journal of Physical Education and Sport, vol. 11, 2011, 434-437.

[19] W. Fu, Research Advancements in Humanoid Compression Garments in Sports. Int J Adv Robotic Sy, vol. 10, 2012, 66-72.

[20] M. Janzekovic, Polar sport tester for cattle heart rate measurements: Intech Open Access Publisher, 2010, 157172.

[21] M. Felix and A. G. Dimitriu, Orginal study the effects of physical training over QT dispersion and QTC dispersion to the beginner sportsmen. Fascicula, vol.2, 2011, 105-110.

[22] M. C. Mandyam, A Longer Qt Interval is Associated with Increased Risk of Atrial Fibrillation. Circulation, vol. 126, 2012, A16639.

[23] I. Goldenberg, QT interval: how to measure it and what is" normal". Journal of cardiovascular electrophysiology, vol. 17, 2006, 333-336.

[24] L. Salahuddin, Ultra short term analysis of heart rate variability for monitoring mental stress in mobile settings. Proc. $29^{\text {th }}$ IEEE. Engineering in Medicine and Biology Society, 2007. 4656-4659.

[25] M. W. Driller, The effects of wearing lower body compression garments during a cycling performance test. Int J Sports Physiol Perform, vol. 8, 2013, 300-306.

[26] D. R. Hooper, Roles of an Upper-Body Compression Garment on Athletic Performances. Journal of Strength \& Conditioning Research, Vol. 29, 2015, 2655-2660. 